\title{
Erratum: Model for Plane Turbulent Couette Flow [Phys. Rev. Lett. 75, 2956 (1995)]
}

Peter W. Egolf and Daniel A. Weiss

(Received 3 October 2016; published 9 March 2017)

DOI: 10.1103/PhysRevLett.118.109903

Three formulas in this article show a misprint. On the other hand, all the figures were calculated with the correct equations, and, therefore, remain unaltered. In the remainder these three equations are listed in their corrected forms.

First, Eq. (11) should read as

$$
g_{1}(\eta)=\frac{1}{2}\left\{1+\sqrt{\frac{4-\beta}{\beta}} \tan \left[\arctan \left(\sqrt{\frac{\beta}{4-\beta}}\right) \eta\right]\right\}
$$

Next, Eq. (12) should read as

$$
\alpha=\left[\sqrt{(4-\beta) / \beta} / \arctan \sqrt{\frac{\beta}{4-\beta}}\right] \frac{\beta}{2} .
$$

Finally, Eq. (15) should read as

$$
g_{21}=-\beta g_{1}\left(1-g_{1}\right)=-\frac{\beta}{4}\left\{1-\left(\frac{4-\beta}{\beta}\right) \tan ^{2}\left[\arctan \left(\sqrt{\frac{\beta}{4-\beta}}\right) \eta\right]\right\} .
$$

